\title{
Sensitive Voltammetric Analysis of Cetirizine Using Electrochemical Sensor Based on Poly (methyl orange) Modified Carbon Nanotube Paste Electrode
}

\author{
Mohammed Monirul Islam ${ }^{1 *}$, Noor E Hafsa ${ }^{2}$, Muhammad Muhitur Rahman ${ }^{3}$, \\ MD Arifuzzaman ${ }^{4}$, and Sayeed Rushd \\ ${ }^{1}$ Department of Biomedical Sciences, College of Clinical Pharmacy, King Faisal University, \\ Al-Ahsa, 31982, Saudi Arabia \\ ${ }^{2}$ Department of Computer Science, College of Computer Science And Information Technology, King \\ Faisal University, Al-Ahsa, 31982, Saudi Arabia \\ ${ }^{3}$ Department of Civil and Environmental Engineering, College of Engineering, King Faisal University, \\ Al-Ahsa, 31982, Saudi Arabia \\ ${ }^{4}$ Department of Civil and Environmental Engineering, College of Engineering, King Faisal University, \\ Al-Ahsa, 31982, Saudi Arabia \\ ${ }^{5}$ Department of Chemical Engineering, College of Engineering, King Faisal University, Al-Ahsa, \\ 31982, Saudi Arabia \\ *E-mail: mislam@kfu.edu.sa
}

Received: 2 xxx 2021 / Accepted: 15 December 2021 / Published: 2 February 2022

\begin{abstract}
Poly (methyl orange) was demonstrated as an effective polymer for the modification of electrode surface for the electrochemical investigation of various drug molecules. An efficient detection of cetirizine (CTZ), a renowned antihistamine drug is of great importance. Systematic analysis of CTZ was done through cyclic voltammetry $(\mathrm{CV})$ method in phosphate buffer saline with $\mathrm{pH} 7.0$ at $0.1 \mathrm{~V} / \mathrm{s}$ scan rate. The developed poly(methyl orange) modified carbon nanotube electrode (PMO/CNTPE) was highly conductive and it was demonstrated through $\mathrm{CV}$ and electrochemical impedance spectroscopy methods. Under best possible investigational circumstances, the developed non-enzymatic sensing device was proficient to detect $\mathrm{CTZ}$ with a broad concentration range $(2 \mu \mathrm{M}$ to $45 \mu \mathrm{M})$ and produced lower limit of detection $(0.076 \mu \mathrm{M})$. Additionally, the designed electrochemical sensor exhibited superior stability along with excellent repeatability and reproducibility showcasing its efficient sensing ability. The fabricated sensing device was inspected to check its applicability in the analysis of medicinal formulations with acceptable recovery.
\end{abstract}

Keywords: Cetirizine, poly(methyl orange), multi walled carbon nanotubes, tablet sample analysis $\underline{\text { FULL TEXT }}$ 
(C) 2022 The Authors. Published by ESG (www.electrochemsci.org). This article is an open access article distributed under the terms and conditions of the Creative Commons Attribution license (http://creativecommons.org/licenses/by/4.0/). 